\title{
Faecal bifidobacteria concentrations in intensive care unit patients receiving enteral nutrition for different lengths of time
}

\author{
H. A. Majid ${ }^{1}$, P. W. Emery ${ }^{1}$, R. J. Beale ${ }^{2}$, T. Sherry $\mathrm{T}^{2}$ and K. Whelan ${ }^{1}$ \\ ${ }^{1}$ Nutritional Sciences Division, King's College London, London SE1 9NH, UK and ${ }^{2}$ Intensive Care Unit, Guy's and \\ St Thomas' NHS Foundation Trust, London SE1 7EH, UK
}

The gastrointestinal microbiota are involved in inhibiting enteropathogenic colonization, stimulating immunity and producing short-chain fatty acids that stimulate colonic water absorption. Dysbiosis of the gastrointestinal microbiota can occur during enteral nutrition (EN) and patients with diarrhoea during EN have lower faecal concentrations of bifidobacteria ${ }^{(1)}$. Moreover, we recently found that faecal concentrations of bifidobacteria in patients who had been receiving exclusive EN for at least 12 days were lower than the values reported normally for healthy subjects ${ }^{(2)}$. In order to investigate the time course over which low bifidobacterial concentrations may develop, we have now compared these results with baseline values from patients who are being recruited to an intervention study, who have been on EN for a shorter time.

All patients were adults being treated on an intensive care unit and receiving exclusive EN with a fibre and fructo-oligosaccharides (FOS)-enriched formula. One group had received EN for 2-6 (median 5) days and another group had received EN for 14 days. A faecal sample was collected from each patient and the microbiota analysed using fluorescent in situ hybridisation, with the probes to detect bifidobacteria (Bif164), lactobacilli (Lab158), clostridia (EREC482) and bacteroides (Bac303) while total cell numbers were quantified using 4, 6-diamidino-2-phenylindole for nucleic acid staining. Statistical analysis was by Mann-Whitney $U$ or Student's $t$-test, according to the sample distribution. Any differences were considered significant when $P<0.05$.

Mean (SD) age for those in the 2-6-day group was $71.1(10.3)$ years and in the 14-day group was $63.4(14.1)$ years $(P=0.165)$. The concentration of bifidobacteria, but not the other bacterial species was lower in the day $2-6$ group than the day 14 group (see Table). This was despite there being no difference in the amount of prebiotic FOS being delivered to patients in the $2-6$ day group $(6.4 \pm 1.1 \mathrm{~g} / \mathrm{d})$ versus the 14 day group $(7.1 \pm 0.9 \mathrm{~g} / \mathrm{d})(P=0.117)$. In addition, there was no significant difference in the number of antibiotics prescribed to patients in the 2-6 day group (median 3, range 1-6) and the 14 day group (median 3, range 0-5) $(P=0.76)$.

\begin{tabular}{|c|c|c|c|}
\hline \multirow{2}{*}{$\begin{array}{l}\text { Microbiota concentration }\left(\log _{10} \text { per g dry faeces); }\right. \\
\text { median ( } \min -\max )\end{array}$} & \multicolumn{2}{|c|}{ Days } & \multirow[b]{2}{*}{$P$ value } \\
\hline & $2-6(n=9)$ & $14(n=16)$ & \\
\hline Total cells & $10.0(6.0-10.1)$ & $10.0(9.1-10.6)$ & 0.978 \\
\hline Bacteroides & $9.5(6.0-10.0)$ & $9.7(6.0-10.4)$ & 0.598 \\
\hline Clostridia & $8.8(6.0-9.5)$ & $8.8(7.4-10.1)$ & 0.718 \\
\hline Lactobacilli & $7.8(6.0-8.9)$ & $8.1(6.0-9.4)$ & 0.598 \\
\hline Bifidobacteria & $8.6(6.0-9.3)$ & $7.1(6.0-8.9)$ & $0.017 *$ \\
\hline
\end{tabular}

It appears that faecal bifidobacteria concentrations were lower in patients who had been enterally fed for longer. It will be important to confirm this result with longitudinal studies. The cause may relate to a variety of factors such as the severity and duration of the patient's disease and the length of antibiotic treatment as well as the period of enteral feeding.

1. Whelan K, Judd PA, Tuohy KM, et al. (2009) Am J Clin Nutr 89, 240-247.

2. Majid HA, Emery PW \& Whelan K (2007) J Hum Nutr Diet 20, 364. 